\title{
Guangdong's Study of the Effectiveness of China's Inactivated Vaccines Against the SARS-CoV-2 B.1.617.2 (Delta) Variant
}

\author{
Fuzhen Wang'; Zhijie An'; Lance Rodewald'; Dan Wu'; Lin Tang'; \\ Hui Zheng'; Qianqian Liu'; George F. Gao'; Zundong Yin ${ }^{1, *}$
}

Guangdong CDC published in preprint a critically important study of the effectiveness of China's inactivated vaccines against the B.1.617.2 (Delta) variant of severe acute respiratory syndrome coronavirus 2 (SARS-CoV-2) - a strain of the virus that causes coronavirus disease 2019 (COVID-19) and is circulating globally (1-2). The Delta variant managed to get through China's strong international border quarantine protection and spark an outbreak in Guangdong Province in May-June 2021. While the outbreak was being stopped using guidance of the Protocol for Prevention and Control of COVID-19 (3), the investigators took advantage of provisions in the Protocol that identified close contacts of anyone infected with SARS-CoV-2, quarantined the close contacts, and tested them for infection while in managed quarantine. Guangdong CDC determined the vaccination status of these close contacts and which of the close contacts became infected, allowing an estimate of the effectiveness of the vaccines used in Guangdong against COVID-19 caused by the Delta variant. The study design was elegant, as the investigators were able to evaluate vaccine effectiveness (VE) in the population with the highest force of infection in the outbreak area, maximizing the possibility of making an accurate estimate of VE.

\section{THE FINDINGS}

The investigators found that the inactivated vaccines provide excellent protection from pneumonia and severe illness caused by the Delta variant — results that are consistent with the Phase III efficacy clinical trials against the original SARS-CoV-2 prototype. Unadjusted and adjusted VE estimates for full vaccination (14 days after the second dose with a $21+$ day interval between two doses) against pneumonia among individuals 18 years and above were $78 \%$ [95\% confidence interval (CI): 45\%-91\%] and 70\% (95\%
CI: 43\%-96\%), respectively, with 100\% VE against severe illness. Partial vaccination, in contrast, showed little effectiveness against pneumonia — unadjusted and adjusted VEs of partial vaccination were $1.4 \%$ and $8.4 \%$, respectively. Importantly, there were no severe or critical breakthrough cases, while unvaccinated close contacts had 19 severe or critical cases.

\section{IMPORTANCE OF THE STUDY}

As the COVID-19 pandemic continues, SARSCoV-2 evolves in unpredictable directions, with emerging variants that differ in transmissibility, infectiousness by age group, and severity of illness. Since vaccines are essential tools for pandemic control, it is vital that their performance is continuously assessed. Guangdong CDC's study is an excellent example of extracting valuable information on the realworld performance of the China-produced inactivated COVID-19 vaccines. It is the first real-world VE study of inactivated vaccines against the Delta variant. Their study shows the feasibility of monitoring VE against incoming variants, even though the number of infections in China remains extremely low. Since the two most prominent inactivated COVID-19 vaccines used in China are approved and recommended for emergency use by the World Health Organization (WHO) and are in widespread use globally, this study provides some assurance to the global scientific community that the vaccines hold up against the Delta variant.

\section{RELEVANCE TO CHINA'S COVID-19 VACCINATION STRATEGY}

Guangdong's VE study provides some assurance that China's current COVID-19 vaccination strategy remains on track. The current phase of the strategy 
includes vaccination of older people, who have the highest risk of severe illness from SARS-CoV-2 infection. Since there is an ever-present risk of importation, it is useful to know that the vaccines reduce risk severe illness and death. Knowing that the vaccines retain effectiveness against the current variant provides some confidence for expanding vaccinations to adolescents, $12-17$ years of age, to help build wholepopulation immunity. Guangdong's study serves as a good example for other researchers in China to conduct VE evaluations when managing a local epidemic. A growing evidence base will help keep the strategy on track.

The study reinforces that it takes two doses of the inactivated vaccines to protect. Individuals who completed the first dose should receive their second dose as soon as possible, in accordance with technical vaccination recommendations for COVID-19 vaccines in China.

\section{VACCINATION PROGRESS}

To date, COVID-19 vaccines from 7 manufacturers have been approved for conditional marketing or emergency use in China. From Phase III clinical trials of the 3 conditionally approved inactivated vaccines and the adenovirus vectored vaccine, we know that protective efficacy ranges from $50 \%$ to $78 \%$. The other 3 COVID-19 vaccines are approved for emergency use, and include a recombinant subunit vaccine [made with Chinese hamster ovary $(\mathrm{CHO})$ cells] and 2 inactivated vaccines. These three have shown good immunogenicity and safety based on the Phase II clinical trial results and emergency-use adverse event following immunization (AEFI) surveillance.

China's COVID-19 vaccination campaign was officially launched on December 15, 2020, with the initial target population of 18-59 years old in occupations at high risk of infection, subsequently expanding to everyone 18 years and older. As of August 11, 2021, more than 1.82 billion doses of COVID-19 vaccines have been administered in China. Almost 770 million people are fully vaccinated, achieving a national coverage rate of $55 \%$, on par with high- or middle-income countries such as Chile, Canada, Germany, Italy, the United Kingdom, and the United States that have full-vaccination population coverage levels between $50 \%$ and $68 \%$ (4).

\section{LOOKING FORWARD}

From the very beginning of this pandemic, research and evaluation have been critically important to understand and control the disease (5). The Guangdong VE study is reassuring, but it is only one of hundreds to thousands of evaluations needed to monitor and adjust pandemic control strategies. Vaccination strategy is complex and necessarily dynamic because of continuous virus evolution and changes in COVID-19 epidemiology. As new vaccines are developed and new variants emerge, real world vaccine performance must be monitored. The most commonly used COVID-19 vaccines globally are inactivated, mRNA, and adenovirus-vectored vaccines. The effectiveness rates of BNT162b2 and ChAdOx1 CoVID-19 vaccine (after two doses for both vaccines) were $88.0 \%$ (95\% CI: 85.3 to 90.1) and 67.0\% (95\% CI: 61.3 to 71.8$)$, respectively, among those with the Delta variant (6). Thus far, and with the Guangdong study included, they are all showing high effectiveness against the Delta variant.

Immunization programs have considerable experience monitoring vaccine coverage and uptake, vaccine safety, confidence in vaccines and immunization, and vaccine effectiveness. All of the assets of China's immunization programs, national and provincial, in collaboration with immunization programs in other countries and international organizations like the WHO, United Nations Children's Fund (UNICEF), The Global Alliance for Vaccines and Immunisation (GAVI), and Coalition for Epidemic Preparedness Innovations (CEPI), will be needed to manage this unpredictable pandemic.

Conflicts of interest: No conflicts of interest were reported.

Funding: Supported by COVID-19 Vaccines Scientific Research Project of the China National Key R\&D Program "Post-marketing Research on Immunity Persistence and Effectiveness of Inactivated COVID-19 Vaccine" (Grant Number: 2021YFC 0863900).

\section{doi: $10.46234 / \mathrm{ccdcw} 2021.179$}

\# Corresponding author: Zundong Yin, yinzd@chinacdc.cn.

${ }^{1}$ Chinese Center for Disease Control and Prevention, Beijing, China.

Submitted: August 13, 2021; Accepted: August 16, 2021 


\section{REFERENCES}

1. Kang M, Yi Y, Li Y, Sun LM, Deng AP, Hu T, et al. Effectiveness of inactivated COVID-19 vaccines against COVID-19 pneumonia and severe illness caused by the B.1.617.2 (Delta) variant: evidence from an outbreak in Guangdong, China. http://dx.doi.org/10.2139/ssrn. 3895639. [2021-8-5].

2. World Health Organization. Tracking SARS-CoV-2 variants. https://www. who.int/en/activities/tracking-SARS-CoV-2-variants/. [2021-8-12].

3. Liu FF, Zheng CJ, Wang LP, Geng MJ, Chen H, Zhou S, et al. Interpretation of the protocol for prevention and control of COVID-19 in China (Edition 8). China CDC Wkly 2021;3(25):527-30 http://dx.doi.org/10.46234/ccdcw2021.138.

4. Share of the population fully vaccinated against COVID-19. OurWorldInData.org. https://ourworldindata.org/grapher/share-peoplefully-vaccinated-covid. [2021-8-12].

5. Wang C, Horby PW, Hayden FG, Gao GF. A novel coronavirus outbreak of global health concern. Lancet 2020;395(10223):470 - 3 . http://dx.doi.org/10.1016/S0140-6736(20)30185-9.

6. Bernal JL, Andrews N, Gower C, Gallagher E, Simmons R, Thelwall S, et al. Effectiveness of Covid-19 vaccines against the B.1.617.2 (delta) variant. N Engl J Med 2021;385(7):585 - 94. http://dx.doi.org/10. 1056/NEJMoa2108891. 